\title{
Análise florística e estrutural de sistemas silviagrícolas em Tomé-Açu, Pará
}

\author{
Édson Luis Bolfe ${ }^{(1)}$ e Mateus Batistella ${ }^{(1)}$ \\ (1)Embrapa Monitoramento por Satélite, Avenida Soldado Passarinho, № 303, CEP 13070-115 Campinas, SP. E-mail: bolfe@cnpm.embrapa.br, \\ mb@cnpm.embrapa.br
}

Resumo - O objetivo deste trabalho foi analisar a composição florística e estrutural de sistemas silviagrícolas em Tomé-Açu, Pará. Os dados dendrométricos foram obtidos por inventário em 40 parcelas amostrais, com três unidades cada uma, no total de 120 unidades de 10x10 m. Foi inventariada a média de 1.424,3 indivíduos por hectare, pertencentes a 27 famílias e a 54 espécies. Tendo-se considerado a variabilidade dos estágios vegetativos, os diferentes sistemas silviagrícolas (SAF) foram divididos em quatro classes hierárquicas: SAF 1, SAF 2, SAF 3, e SAF 4, para estabelecer um sistema de classificação passível de ser utilizado em outras avaliações de campo e em classificações digitais por meio do sensoriamento remoto. Espécies observadas em outros sistemas da região amazônica também foram relevantes para este estudo, especialmente Theobroma cacao, T. grandiflorum e Euterpe oleracea que, juntas, apresentaram médias de frequência relativa de $51 \%$, densidade relativa de $69,2 \%$, dominância relativa de $50,1 \%$ e índice de valor de importância de $56,8 \%$. Os dados médios de diversidade florística, abundância, área basal e valor de importância indicam os sistemas silviagrícolas da região de Tomé-Açu como sistemas de produção com potencial econômico e ambiental, se adotado manejo adequado e racional.

Termos para indexação: agroecologia, Amazônia, integração lavoura-floresta, sistemas agroflorestais, uso da terra.

\section{Floristic and structural analysis of agroforestry systems in Tomé-Açu, Pará, Brazil}

\begin{abstract}
The objective of this work was to analyze the structural and floristic composition of agroforestry systems in Tomé-Açu, Pará, Brazil. Dendrometric data were obtained through an inventory of 40 sampling plots, with three sampling units each, comprising 120 sampling units with $10 \times 10 \mathrm{~m}$ each. The average of 1,424.3 individuals per hectare belonging to 27 families and 54 species was inventoried. Considering the variability of vegetative stages, the different agroforestry systems (SAF) were divided into four hierarchical classes: SAF 1, SAF 2, SAF 3, and SAF 4, to set an classification system which can be used in other field evaluations and in digital classifications developed by means of remote sensing. Species observed in other agroforestry systems in the Amazon region were also relevant to this work, specially Theobroma cacao, Theobroma grandiflorum and Euterpe oleracea which, together, showed averages of $51 \%$ relative frequency, $69.2 \%$ relative density, $50.1 \%$ relative dominance, and $56.8 \%$ importance value index. The average data of the floristic diversity, abundance, basal area, and importance value index indicate that the agroforestry systems in the Tomé-Açu region are production systems with economic and environmental potential, if appropriate and rational management is adopted.
\end{abstract}

Index terms: agroecology, Amazonia, crop-forest integration, agroforestry systems, land use.

\section{Introdução}

A região Amazônica é composta por um mosaico de paisagens, definido tanto pela heterogeneidade dos ecossistemas existentes quanto pela crescente intensificação agropecuária, refletida no uso da terra, na ocupação humana e no desenvolvimento local (Batistella et al., 2008). O espaço amazônico pode ser visto como um espaço segmentado e organizado de acordo com agentes predominantes de ocupação, que assumem diferentes identidades socioeconômicas e institucionais (Godfrey \& Browder, 1996). Assim, o conhecimento relacionado aos diferentes usos e coberturas da terra dessa região é relevante para subsidiar os processos de tomada de decisão para o planejamento regional, o que evita simplificações e generalizações, pois considera as especificidades locais. Nesse sentido, os sistemas que integram lavoura-pecurária-floresta 
estão sendo amplamente estudados, com ênfase em sistemas no binômio lavoura-floresta, ou seja, sistemas agroflorestais ou silviagrícolas (Osterroht, 2002; Santiago, 2004; Carvalho, 2006; Kato et al., 2006; Luizão et al., 2006; Castro et al., 2009).

Valois (2003) observa que os sistemas que integram lavoura e floresta na Amazônia têm demostrado vantagens em relação aos monocultivos, pois além de evitar a degradação de solos e proporcionar o aumento da matéria orgânica, com consequente benefício para a melhoria química e física dos solos, essa associação de culturas reduz a incidência de pragas e doenças. $\mathrm{O}$ autor destaca, ainda, que esse sistema não constitui apenas um sistema de produção, mas um sistema de uso da terra de maneira estratégica, pois a produção por unidade de área pode ser elevada, principalmente com a integração, simultânea ou escalonada, de espécies anuais, semiperenes, perenes, madeireiras e não madeireiras, potencialmente compatíveis com os padrões dos produtores rurais familiares.

Após analisar os ciclos biogeoquímicos em sistemas silviagrícolas da Amazônia, Luizão et al. (2006) concluíram que esses sistemas, compostos de espécies nativas de fruteiras e árvores madeireiras, mostraram-se eficientes para a reutilização de áreas abandonadas ou degradadas, pois recuperam não apenas a capacidade produtiva da terra, para alimentos e madeira, mas também diversos serviços ambientais, tais como, a fixação de carbono na biomassa, a circulação da água na interface solo-planta-atmosfera e a reciclagem de nutrientes minerais. Os autores recomendam, ainda, que a integração lavoura-floresta seja direcionada especialmente para a recuperação deáreas já desmatadas e abandonadas na Amazônia brasileira.

Porém, cada sistema silviagrícola implantado tem uma dinâmica de crescimento vegetativo diferenciado devido às condições edafoclimáticas locais e à integração de espécies anuais, semiperenes e perenes utilizadas na composição das áreas de cultivo, que formam classes distintas de produção. Assim, estudos sobre a composição florística e estrutural dos diferentes sistemas são relevantes e constituem a primeira etapa para as avaliações sobre o habitat e a diversidade. Tais estudos sãonecessáriospara amparar decisõesgerenciais vinculadas às atividades econômicas e ambientais, como políticas públicas de uso dos recursos naturais, manejo florestal, estudos de ciclagem de nutrientes, estimativa de biomassa e carbono, zoneamentos e monitoramentos geoespaciais.
Tomé-Açu teve seu desenvolvimento rural a partir da década de 1920, com o início da imigração japonesa. No início, os imigrantes implantaram a horticultura e depois a cultura da pimenta-do-reino (Piper nigrum L.), com apoio do governo brasileiro, o que possibilitou o avanço tecnológico e tornou o Pará o maior produtor de pimenta-do-reino do país. Com o declínio do ciclo da pimenta-do-reino, a partir da década de 1970, causado principalmente por questões fitossanitárias (fusariose), os agricultores buscaram novas alternativas de produção. Segundo Homma (2003), a solução dos imigrantes para essa crise foi a diversificação das atividades, com destaque para a pecuária e a fruticultura, em especial de mamão 'Hawaí', melão, acerola, laranja, dendê, açaí, cacau, cupuaçu, maracujá, entre outras fruteiras nativas e exóticas, o que gerou um novo ciclo econômico para a região. A diversificação das culturas foi associada a um novo sistema de produção, desenvolvido a partir de inúmeras experimentações locais, que geraram arranjos produtivos com diferentes espécies e promoveram a inserção da produção em novos mercados.

Os sistemas silviagrícolas atuais, implantados entre 1 e mais de 30 anos, têm grande variedade de espécies anuais, frutíferas e madeireiras. Homma (1998) destaca que o sucesso do desenvolvimento rural da região deve-se à elevada experiência dos agricultores nipo-brasileiros, à sua mentalidade inovadora, à visão holística de mercados futuros e ao espírito associativista que permitiu a criação, em 1931, da Cooperativa Agrícola Mista de Tomé-Açu (Camta), inicialmente estabelecida para vender hortaliças e que atualmente comercializa os produtos desses sistemas (frutos, polpa, suco e óleos) para diversos países.

O objetivo deste trabalho foi avaliar a composição florística e estrutural de sistemas silviagrícolas em propriedades rurais de Tomé-Açu.

\section{Material e Métodos}

O trabalho foi realizado no Distrito de Quatro Bocas (2²5'09" S , 4809'10" W), Município de Tomé-Açu, na Mesorregião Nordeste Paraense (Instituto Brasileiro de Geografia e Estatística, 2008). O relevo é caracterizado por baixos platôs aplainados (tabuleiros), terraços e várzeas, que variam entre 14 e $96 \mathrm{~m}$ de altitude. Ocorrem os solos classificados como LatossoloAmarelo distrófico com textura argilosa média, Plintossolos, Gleissolos e Neossolos Flúvicos eutróficos e distróficos em 
associação (Rodrigues et al., 2001). A vegetação original é composta por Floresta Ombrófila Densa das Terras Baixas, atualmente bastante alteradas e que ensejam a ocorrência de florestas secundárias. Remanescentes florestais são observados principalmente às margens dos cursos d'água. A drenagem é representada pela Bacia do Rio Acará-Mirim, que nasce ao sul do município, toma a direção norte-nordeste e deságua no Rio Acará. A região de Tomé-Açu tem clima mesotérmico e úmido, do tipo Ami de acordo com a classificação de Köppen, com temperatura média anual de $26^{\circ} \mathrm{C}$ e umidade relativa do ar em torno de $85 \%$. A média anual de precipitação é de $2.300 \mathrm{~mm}$. Apesar de regulares, as chuvas não se distribuem igualmente durante o ano, e a média mensal varia entre 54 em agosto e $440 \mathrm{~mm}$ em março. No trimestre mais chuvoso, fevereiro, março e abril, as médias mensais ultrapassam $300 \mathrm{~mm}$ e, no trimestre menos chuvoso, agosto, setembro e outubro, as médias ficam próximos de $60 \mathrm{~mm}$ (Pacheco \& Bastos, 2008).

A partir da imagem do satélite Landsat 5/TM de 2005, foi identificada a distribuição de 217 lotes de agricultores da região, potenciais para os levantamentos de campo (sócios e não sócios da Camta). Destes, foram selecionados de forma aleatória 40 lotes que representam as parcelas amostrais. As parcelas foram constituídas por três unidades cada, no total de 120 unidades amostrais compostas de quadrados de $10 \times 10 \mathrm{~m}, 3 \times 3 \mathrm{~m}$ e $1 \times 1 \mathrm{~m}$.

Nas unidades amostrais de 10x10 m, foi estimada a altura total $(\mathrm{H})$ e mensurado o diâmetro à altura do peito (DAP), se igual ou superior a $10 \mathrm{~cm}$, para todos os indivíduos identificados em campo e classificados com base em informações da Lista das Espécies da Flora do Brasil (Forzza et al., 2010) e The Angiosperm Phylogeny Group (2009). Nas subunidades de 3x3 m, esses mesmos parâmetros foram obtidos para os indivíduos com DAP entre 2,5 e $10 \mathrm{~cm}$. Nas subunidades de $1 \times 1 \mathrm{~m}$, foram identificados e quantificados todos os indivíduos existentes. As coordenadas geográficas das unidades amostrais foram obtidas por meio de sistema de posicionamento global (GPS). Foram coletadas informações, por meio de entrevistas, sobre o histórico de uso e ocupação das áreas, a idade e as espécies inicialmente implantadas nos SAF. Como informação adicional, estimou-se visualmente a percentagem da cobertura de copa, cobertura de herbáceas, de liteira e solo exposto nas unidades amostrais.

Para a análise estrutural da vegetação arbórea dos SAF estudados, foram adotadas recomendações propostas por Brower et al. (1998), tendo-se considerado os índices de: abundância absoluta $(\mathrm{Na})$, abundância por hectare $(\mathrm{N})$, frequência absoluta $(\mathrm{Fa})$, frequência relativa $(\mathrm{Fr})$, área basal $(\mathrm{G})$, densidade relativa (Dr), dominância relativa (Dor) e índice de valor de importância (IVI).

A diversidade florística foi calculada pelos índices de Simpson $\left(\mathrm{S}^{\prime}\right)$ e de Shannon $\left(\mathrm{H}^{\prime}\right)$. O índice de Simpson varia na escala de 0 a 1 e, quanto mais alto, maior a probabilidade de os indivíduos serem da mesma espécie, ou seja, maior a dominância e menor a diversidade (Magurran, 1988). O índice de Shannon, normalmente, tem seu valor entre 1,5 e 3,5, é raro que ultrapasse 4,5, e é máximo se existir igualdade entre o número de espécies (Shannon, 1948). Foi calculada, ainda, a uniformidade de distribuição dos indivíduos pelo índice de equabilidade de Pielou ( $\left.\mathrm{J}^{\prime}\right)$. O valor de $\mathrm{J}^{\prime}$ varia entre 0 e 1 , em que 1 é a igualdade na abundância de todas as espécies amostradas (Magurran, 1988). Para analisar a similaridade florística qualitativa entre as diferentes classes silviagrícolas, foi aplicado o índice de Sörensen $\left(\mathrm{IS}_{\mathrm{s}}\right)$. O valor de $\mathrm{IS}_{\mathrm{s}}$ varia de 0 para nenhuma similaridade a 1 para similaridade total, e IS $_{\mathrm{s}}<0,50$ representa baixa similaridade florística (Brower et al., 1998). Para os referidos cálculos utilizou-se o sistema Bio-Dap (Magurran, 1988).

\section{Resultados e Discussão}

Nos sistemas estudados, foram inventariados 5.697 indivíduos com DAP superior a 2,5 cm (média de 1.424,3 indivíduos por hectare), pertencentes a 54 espécies de 27 famílias botânicas. Esses números são semelhantes aos encontrados por Santos et al. (2004), que observaram a ocorrência de 27 famílias e 61 espécies, e por Ribeiro et al. (2004), que encontraram 26 famílias e 59 espécies, em sistemas silviagrícolas da região do Município de Cametá, PA. Porém tais números são superiores às 18 famílias e 28 espécies observadas por Vieira et al. (2007) ao analisar sistemas similares em Igarapé-Açu, PA.

Smith et al. (1998) estudaram sistemas silviagrícolas em quatro estados da Amazônia e observaram a existência de diversos modelos implantados, dos quais os analisados contêm somente de duas a seis espécies. Em revisão de literatura sobre sistemas agroflorestais na Amazônia Brasileira, Brienza Júnior et al. (2009) constataram que os trabalhos publicados tratam de poucas espécies ao mesmo tempo. Em termos cumulativos, $60 \%$ da literatura utilizaram seis espécies. Entretanto, o número de trabalhos com 15 ou mais 
espécies foi cerca de 5\%. A constatação de poucos trabalhos com muitas espécies reflete a dificuldade de manejo, em um único sistema de produção. Em estudos realizados no vale do rio Acre, Nobre (1998) destaca que os agricultores desenvolvem sistemas de integração lavoura-floresta orientados principalmente para a geração de renda, geralmente caracterizados pelo consórcio de espécies, para atender o mercado consumidor. Assim, observa-se que estes SAF possuem número variável de espécies, com diferentes potencialidades mercadológicas.

A partir da análise dos dados e parâmetros da estrutura da vegetação obtidos em campo foi possível definir um esquema que diferenciou quatro classes de sistemas silviagrícolas (SAF 1, SAF 2, SAF 3 e SAF 4) para a região de Tomé-Açu (Tabela 1).

Nos sistemas analisados, as famílias com maior riqueza em espécies foram: Fabaceae (dez espécies), Meliaceae (cinco espécies); Bignoniaceae (quatro espécies); Annonaceae, Anacardiaceae e Rubiaceae (três espécies), que juntas representam $54 \%$ das espécies amostradas (Tabela 2). Quatro espécies foram comuns a todos os SAF: Theobroma cacao e Theobroma grandiflorum Schum., também destacadas por Mendes (2003) ao quantificar economicamente a contribuição dos sistemas silviagrícolas de Tomé-Açu, e Euterpe oleracea Mart. e Cecropia palmata Willd. As espécies de maior abundância absoluta foram T. cacao L., E. oleracea, T. grandiflorum e Hevea brasiliensis (Willd. ex A. Juss.) Müll. Arg., com 671, 305, 228 e 112 indivíduos, respectivamente, que representaram, em conjunto $77 \%$ de todos os indivíduos amostrados. Ribeiro et

Tabela 1. Dados e parâmetros dos sistemas silviagrícolas de Tomé-Açu, PA, $2008^{(1)}$.

\begin{tabular}{lccccc}
\hline Dados/Parâmetros & \multicolumn{4}{c}{ Classificações silviagrícolas } & \multirow{2}{*}{ Média } \\
\cline { 2 - 5 } & SAF 1 & SAF 2 & SAF 3 & SAF 4 & \\
\hline Número de famílias & 8 & 15 & 14 & 25 & 15,8 \\
Número de espécies & 9 & 19 & 19 & 40 & 21,8 \\
N (ni ha $\left.^{-1}\right)$ & 747 & 1.770 & 1.723 & 1.457 & $1.424,3$ \\
C (\%) & 29,5 & 45 & 63 & 78 & 53,9 \\
DAP médio (cm) & 5,2 & 6,4 & 10,7 & 13,7 & 9,0 \\
DAP (s) & 3,6 & 3,3 & 6,0 & 11,4 & - \\
DAP (CV, \%) & 68,9 & 50,8 & 56,2 & 83,1 & - \\
G (m ${ }^{2}$ ha $\left.{ }^{-1}\right)$ & 2,3 & 7,2 & 20,4 & 36,3 & 16,5 \\
H média (m) & 2,9 & 3,7 & 5,9 & 7,4 & 5,0 \\
H (s) & 1,4 & 2,0 & 2,9 & 5,4 & - \\
H (CV, \%) & 48,3 & 53,5 & 49,9 & 71,9 & - \\
\hline
\end{tabular}

${ }^{(1)} \mathrm{N}$, abundância; $\mathrm{C}$, cobertura de copa; DAP, diâmetro médio à altura do peito; $\mathrm{G}$, área basal; $\mathrm{H}$ média, altura total média; $\mathrm{H}$ (s), desvio-padrão; $\mathrm{CV}$, coeficiente de variação. al. (2004) e Vieira et al. (2007) também constataram T. grandiflorum e E. oleracea como as mais frequentes em outros sistemas silviagrícolas no Pará. Entre as espécies comuns a todos os sistemas e de maior abundância, as frutíferas merecem destaque. Outros autores já observaram que as áreas de integração lavoura-floresta na Amazônia apresentam principalmente espécies frutíferas em seus arranjos (Homma, 2004; Ribeiro et al., 2004; Vieira et al., 2007). Essa preferência, possivelmente, está relacionada à demanda do mercado consumidor e à segurança alimentar, além dos longos ciclos de produção responsáveis pela garantia de renda continuada.

Na Tabela 3, estão apresentados os parâmetros para a análise estrutural da vegetação de cada SAF. Em média, as espécies mais abundantes foram T. cacao (566 indivíduos por hectare), seguida de E. oleracea (254 indivíduos por hectare), T. grandiflorum (190 indivíduos por hectare) e H. brasiliensis (186 indivíduos por hectare). Juntas, essas espécies representaram densidade relativa de $80,5 \%$ nos SAF 1 , 2,3 e $4(38,4,15,6,15,1$ e $11,3 \%$, respectivamente). A abundância média (1.424 indivíduos por hectare) é inferior aos 3.009 indivíduos por hectare observados nas várzeas do Rio Jubá, por Santos et al. (2004).

A área basal média foi de $16,5 \mathrm{~m}^{2} \mathrm{ha}^{-1}$. O SAF 4 apresentou a maior área basal e o SAF 1, a menor. No SAF 1, a maior área basal foi para T. cacao e T. grandiflorum, em razão da maior frequência dessas espécies. No SAF 2, T. cacao também apresentou a maior área basal e a maior abundância. $H$. brasiliensis teve a maior área basal no SAF 3, apesar de ter abundância inferior à de E. oleracea e T. cacao. No SAF 4, a maior área basal foi de Carapa guianensis Aubl., mesmo com baixa abundância.

Foi observada a média de $50,1 \%$ de dominância relativa, para os SAF, de três espécies (T. cacao, T. grandiflorum e E. oleracea). A maior dominância do cacaueiro (média de $24,9 \%$ ) deve-se especialmente à implantação de extensas áreas com esta espécie na região, a partir da década de 1980. Euterpe oleracea $(14,7 \%)$ e $T$. grandiflorum (10,5\%), foram implantadas principalmente na década de 1990.

As demais 51 espécies representam 49,9\% da dominância. Entre elas, é relevante a elevada dominância, em alguns SAF, de Annona muricata L. no SAF 1, Gliricidia sp. e Bertholletia excelsa Bonpl. (7,36\%) no SAF 2, H. brasiliensis no SAF 3 e $C$. guianensis no SAF 4, implantadas em diferentes anos. 
Tabela 2. Ocorrência das famílias e espécies nos sistemas silviagrícolas de Tomé-Açu, PA, 2008.

\begin{tabular}{|c|c|c|c|c|c|c|c|}
\hline \multirow[t]{2}{*}{ Família } & \multirow[t]{2}{*}{ Nome científico } & \multirow[t]{2}{*}{ Nome comum } & \multicolumn{4}{|c|}{ Sistema silviagrícola } & \multirow[t]{2}{*}{ Total } \\
\hline & & & SAF 1 & SAF 2 & SAF 3 & SAF 4 & \\
\hline \multirow{3}{*}{ Anacardiaceae } & Mangifera indica $\mathrm{L}$. & Manga & & & & 1 & 1 \\
\hline & Spondias mombin L. & Caja/tapereba & & 1 & & 2 & 3 \\
\hline & Tapirira guianensis Aubl. & Tapiriri & & & & 1 & 1 \\
\hline \multirow{3}{*}{ Annonaceae } & Annona muricata $\mathrm{L}$. & Graviola & 27 & 2 & & & 29 \\
\hline & Rollinia exsucca (DC. ex Dunal) A. DC. & Envira-fofa & & & 1 & 2 & 3 \\
\hline & Xylopia aromatica (Lam.) Mart. & Envira-cana & & & 1 & 2 & 3 \\
\hline Araliaceae & Schefflera morototoni (Aubl.) Maguire, Steyerm. \& Frodin & Morototó & & & & 1 & 1 \\
\hline Arecaceae & Euterpe oleracea Mart. & Açaí & 12 & 98 & 169 & 26 & 305 \\
\hline \multirow{4}{*}{ Bignoniaceae } & Jacaranda copaia (Aubl.) D. Don & Pará-pará & & & 1 & & 1 \\
\hline & Handroanthus albus (Cham.) Mattos & Ipê-branco & & & & 1 & 1 \\
\hline & Tabebuia pentaphylla (L.) Hemsl. & Ipê-rosa & & 1 & & & 1 \\
\hline & Handroanthus serratifolius (Vahl) S.Grose & Ipê-amarelo & & & & 1 & 1 \\
\hline Boraginaceae & Cordia goeldiana Huber & Freijó & & & 6 & 8 & 14 \\
\hline Caryocaraceae & Caryocar villosum (Aubl.) Pers. & Pequiá & 2 & & & 1 & 3 \\
\hline Chrysobalanaceae & Licania kunthiana Hook. f. & Caripé & & & & 3 & 3 \\
\hline \multirow{2}{*}{ Clusiaceae } & Garcinia mangostana $\mathrm{L}$. & Mangostão & & 2 & & 2 & 4 \\
\hline & Platonia esculenta (Arruda) Oken & Bacuri & & & 2 & 14 & 16 \\
\hline Euphorbiaceae & Hevea brasiliensis (Willd. ex A. Juss.) Müll.Arg. & Seringueira & & & 96 & 16 & 112 \\
\hline \multirow{10}{*}{ Fabaceae } & Bowdichia nitida Spruce ex Benth. & Sucupira-amarela & & & & 1 & 1 \\
\hline & Clitoria racemosa G. Don. & Palheteira & & & 3 & 2 & 5 \\
\hline & Derris sp. & Derris & & 13 & & & 13 \\
\hline & Enterolobium schomburgkii (Benth.) Benth. & Fava-de-orelha & & 2 & & & 2 \\
\hline & Erythrina fusca Lour. & Mulungu & & & & 2 & 2 \\
\hline & Erythrina sp. & Eretrina & & & & 1 & 1 \\
\hline & Gliricidia sp. & Gliricídia & & 32 & & 6 & 38 \\
\hline & Inga alba (Sw.) Willd. & Ingá-cordão & & & & 1 & 1 \\
\hline & Schizolobium parahyba (Vell.) S.F. Blake & Paricá & & 3 & 1 & 6 & 10 \\
\hline & Stryphnodendron pulcherrimum (Willd.) Hochr. & Paricazinho & & & & 2 & 2 \\
\hline Flacourtiaceae & Banara guianensis Aubl. & Andorinha & & & 2 & 6 & 8 \\
\hline Lacistemataceae & Lacistema pubescens Mart. & Cafezinho & & & & 1 & 1 \\
\hline \multirow{2}{*}{ Lauraceae } & Licaria puchury-major (Mart.) Kosterm. & Puxuri & & 3 & & & 3 \\
\hline & Persea americana Mill. & Abacate & & 2 & & 2 & 4 \\
\hline Lecythidaceae & Bertholletia excelsa Bonpl. & Castanha-do-pará & & 4 & 2 & 9 & 15 \\
\hline \multirow{2}{*}{ Malvaceae } & Theobroma cacao $\mathrm{L}$. & Cacau & 75 & 256 & 161 & 179 & 671 \\
\hline & Theobroma grandiflorum (Willd. ex Spreng.) K. Schum. & Cupuaçu & 64 & 93 & 53 & 18 & 228 \\
\hline Melastomataceae & Miconia sp. & Papa-terra & & & & 1 & 1 \\
\hline \multirow{5}{*}{ Meliaceae } & Azadirachta indica A. Juss. & Nim & & 1 & & & 1 \\
\hline & Carapa guianensis Aubl. & Andiroba & & & 1 & 25 & 26 \\
\hline & Cedrela odorata $\mathrm{L}$. & Cedro & 1 & & & & 1 \\
\hline & Khaya ivorensis A. Chev. & Mogno-africano & & & 7 & & 7 \\
\hline & Swietenia macrophylla King & Mogno & & & 3 & 6 & 9 \\
\hline \multirow{2}{*}{ Moraceae } & Bagassa guianensis Aubl. & Tatajuba & & & & 1 & 1 \\
\hline & Maquira sclerophylla (Ducke) C.C. Berg & Muiratinga & & & & 1 & 1 \\
\hline Musaceae & Musa paradisiaca $\mathrm{L}$. & Bananeira & 37 & & & & 37 \\
\hline Myristicaceae & Virola surinamensis (Rol. ex Rottb.) Warb. & Virola & & & & 1 & 1 \\
\hline \multirow[t]{2}{*}{ Myrtaceae } & Myrcia sp. & Araçá & 2 & & & & 2 \\
\hline & Alibertia edulis (Rich.) A. Rich. ex DC. & Puruí & & & & 2 & 2 \\
\hline \multirow[t]{2}{*}{ Rubiaceae } & Borreria latifolia (Aubl.) K.Schum. & Rama-verde & & & 1 & & 1 \\
\hline & Coffea arabica $\mathrm{L}$. & Café & & & & 1 & 1 \\
\hline Rutaceae & Citrus medica $\mathrm{L}$. & Limão & & 8 & & 62 & 70 \\
\hline Sapindaceae & Nephelium lappaceum L. & Rambotã & & 1 & & & 1 \\
\hline Urticaceae & Cecropia palmata Willd. & Embaúba & 4 & 2 & 6 & 4 & 16 \\
\hline \multirow[t]{2}{*}{ Verbenaceae } & Tectona grandis L. f. & Teca & & 7 & & 16 & 23 \\
\hline & Não identificada & Não identificada & & & 1 & & 1 \\
\hline Total & & & 224 & 531 & 517 & 437 & 1.708 \\
\hline
\end{tabular}


No SAF 1, as maiores frequências foram de T. cacao, T. grandiflorum, Musa paradisiaca L. e E. oleracea, que representaram, conjuntamente, $81 \%$ do valor total do IVI. No SAF 2, as maiores frequências foram de E. oleracea, T. cacao e T. grandiflorum. As três espécies representam $71,45 \%$ do IVI total. Para o SAF 3, as maiores frequências observadas foram: T. cacao, H. brasiliensis e E. oleracea que representaram, conjuntamente, $76,34 \%$ do IVI total. No SAF 4, as maiores frequencias observadas foram de T. cacao, C. guianensis, B. excelsa, Cordia goeldiana Huber, Swietenia macrophylla King e Cecropia palmata, que representaram $52,11 \%$ do IVI do sistema silviagrícola.

Considerando-se as análises estrutural e florística dos SAF, T. cacao, E. oleracea e T. grandiflorum representaram conjuntamente $51 \%$ da frequência relativa, $69,2 \%$ da densidade relativa, $51,1 \%$ da dominância relativa e $56,8 \%$ do valor total do IVI das 54 espécies observadas nos SAF de Tomé-Açu. O SAF 4 apresentou a maior diversidade de espécies, e o SAF 1 apresentou a maior equabilidade de indivíduos entre as espécies registradas, verificada pelo elevado $\mathrm{J}^{\prime}$ (Tabela 4). O índice de Simpson foi pouco influenciado pela riqueza $(0,23$ para o SAF 1 , com nove espécies, e 0,20 para o SAF 4, com 54 espécies); a densidade das espécies com maior abundância teve maior peso, e o índice foi pouco sensível às espécies que apareceram com apenas um indivíduo na amostra. Ao comparar os valores observados nos SAF em estudo com as médias obtidas por Santos et al. (2004), em sistemas silviagrícolas de várzea do Rio Juba, PA, verificou-se diversidade superior nos SAF da região de Tomé-Açu. A maior diversidade observada nas áreas de estudo, principalmente no SAF 4 (implantado nas décadas de 1980 e 1990), está intimamente ligada a dois fatores. O primeiro está relacionado ao declínio do ciclo da pimenta-do-reino e à implantação dos SAF pelos agricultores nipo-brasileiros, que testaram diversas espécies frutíferas em diferentes arranjos produtivos. Homma (2004) destaca que, a partir da década de 1990, houve avanço da fruticultura, em razão da importância das frutas amazônicas no contexto nacional e internacional. Outro fator, verificado neste

Tabela 3. Parâmetros florísticos e fitossociológicos dos sistemas silviagrícolas de Tomé-Açu, PA, 2008 ${ }^{(1)}$.

\begin{tabular}{|c|c|c|c|c|c|c|c|c|c|c|}
\hline Classe & Nome científico & Nome comum & $\mathrm{Na}$ & $\mathrm{N}\left(\right.$ ni ha $\left.^{-1}\right)$ & $\mathrm{Fa}$ & $\operatorname{Fr}(\%)$ & $\mathrm{G}\left(\mathrm{m}^{2} h \mathrm{a}^{-1}\right)$ & $\operatorname{Dr}(\%)$ & Dor $(\%)$ & IVI \\
\hline \multirow{6}{*}{ SAF 1} & Theobroma cacao L. & Cacau & 75 & 250 & 0,40 & 31,58 & 0,73 & 33,48 & 32,24 & 97,30 \\
\hline & Theobroma grandiflorum (Willd. ex Spreng.) K. Schum. & Cupuaçu & 64 & 213 & 0,23 & 18,42 & 0,73 & 28,57 & 32,49 & 79,49 \\
\hline & Musa paradisiaca $\mathrm{L}$. & Bananeira & 37 & 123 & 0,23 & 18,42 & 0,13 & 16,52 & 5,77 & 40,71 \\
\hline & Annona muricata $\mathrm{L}$. & Graviola & 27 & 90 & 0,07 & 5,26 & 0,21 & 12,05 & 9,46 & 26,78 \\
\hline & Euterpe oleracea Mart. & Açaí & 12 & 40 & 0,13 & 10,53 & 0,21 & 5,36 & 9,61 & 25,50 \\
\hline & Outras espécies (4) & & 9 & 30 & 0,19 & 15,78 & 0,22 & 4,02 & 10,42 & 30,22 \\
\hline Total & & & 224 & 747 & 1,27 & 100,00 & 2,28 & 100,00 & 100,00 & 300,00 \\
\hline \multirow{6}{*}{ SAF 2} & Theobroma cacao L. & Cacau & 256 & 853 & 0,60 & 25,00 & 2,374 & 48,21 & 33,15 & 106,36 \\
\hline & Euterpe oleracea Mart. & Açaí & 98 & 327 & 0,73 & 30,56 & 1,993 & 18,46 & 27,84 & 76,85 \\
\hline & Theobroma grandiflorum (Willd. ex Spreng.) K. Schum. & Cupuaçu & 93 & 310 & 0,17 & 6,94 & 0,478 & 17,51 & 6,68 & 31,14 \\
\hline & Gliricidia sp. & Gliricídia & 32 & 107 & 0,10 & 4,17 & 0,602 & 6,03 & 8,41 & 18,60 \\
\hline & Derris sp. & Derris & 13 & 43 & 0,10 & 4,17 & 0,123 & 2,45 & 1,72 & 8,33 \\
\hline & Outras espécies (14) & - & 39 & 130 & 0,67 & 29,19 & 1,594 & 7,36 & 22,27 & 58,76 \\
\hline Total & & & 531 & 1.770 & 2,40 & 100,00 & 7,16 & 100,00 & 100,00 & 300,00 \\
\hline \multirow{6}{*}{ SAF 3} & Euterpe oleracea Mart. & Açaí & 169 & 563 & 0,47 & 15,93 & 4,27 & 32,69 & 21,01 & 69,62 \\
\hline & Theobroma cacao L. & Cacau & 161 & 537 & 0,90 & 30,72 & 3,49 & 31,14 & 17,16 & 79,01 \\
\hline & Hevea brasiliensis (Willd. ex A. Juss.) Müll. Arg. & Seringueira & 96 & 320 & 0,53 & 18,20 & 8,88 & 18,57 & 43,62 & 80,39 \\
\hline & Theobroma grandiflorum (Willd. ex Spreng.) K.Schum. & Cupuaçu & 53 & 177 & 0,17 & 5,69 & 0,53 & 10,25 & 2,65 & 18,59 \\
\hline & Khaya ivorensis A. Chev. & Mogno-africano & 7 & 23 & 0,10 & 3,41 & 1,22 & 1,35 & 6,00 & 10,77 \\
\hline & Outras espécies (14) & - & 31 & 102 & 0,75 & 26,20 & 1,88 & 5,98 & 9,53 & 41,69 \\
\hline Total & & & 517 & 1.723 & 2,93 & 100,00 & 20,36 & 100,00 & 100,00 & 300,00 \\
\hline \multirow{6}{*}{ SAF 4} & Theobroma cacao L. & Cacau & 179 & 597 & 0,70 & 22,36 & 6,21 & 40,96 & 17,10 & 80,43 \\
\hline & Citrus medica L. & Limão & 62 & 207 & 0,10 & 3,19 & 0,31 & 14,19 & 0,86 & 18,24 \\
\hline & Euterpe oleracea Mart. & Açaí & 26 & 87 & 0,10 & 3,19 & 0,89 & 5,95 & 2,46 & 11,61 \\
\hline & Carapa guianensis Aubl. & Andiroba & 25 & 83 & 0,20 & 6,39 & 7,06 & 5,72 & 19,44 & 31,55 \\
\hline & Theobroma grandiflorum (Willd. ex Spreng.) K. Schum. & Cupuaçu & 18 & 60 & 0,10 & 3,19 & 0,08 & 4,12 & 0,23 & 7,54 \\
\hline & Outras espécies (35) & - & 127 & 421 & 1,87 & 61,65 & 21,62 & 29,09 & 59,88 & 150,74 \\
\hline Total & & & 437 & 1.457 & 3,13 & 100,00 & 36,34 & 100,00 & 100,00 & 300,00 \\
\hline
\end{tabular}

(1) Na, abundância absoluta; N, abundância por hectare; Fa, frequência absoluta; Fr, frequência relativa; G, área basal; Dr, densidade relativa; Dor, dominância relativa; e IVI, índice de valor de importância. 
estudo, está vinculado à diversidade de espécies nos remanescentes florestais do entorno das áreas conduzidas com SAF, fato evidenciado pela observação de diversas espécies nativas de baixa frequência no SAF 4, como a tatajuba (Bagassa guianensis Aubl.), a sucupiraamarela (Bowdichia nitida Spruce ex Benth.), os ipês [Handroanthus albus (Cham.) Mattos), Tabebuia pentaphylla (L.) Hemsl., Handroanthus serratifolius (Vahl) S.Grose], o tapiriri (Tapirira guianensis Aubl.) e a virola [Virola surinamensis (Rol. ex Rottb.) Warb.].

A maior similaridade florística ocorreu entre o SAF 3 e o SAF 4, e a menor entre o SAF 1 e o SAF 4 (Tabela 5). Dos seis pares de SAF analisados, cinco $(83,3 \%)$ apresentaram baixa similaridade $\left(\mathrm{IS}_{\mathrm{s}}<0,5\right)$. A similaridade média entre os SAF foi de $34 \%$, inferior à observada por Santos et al. (2004), que foi de 48,1\%.

A matriz binária baseada no índice de similaridade de Sörensen, elaborada a partir dos levantamentos florísticos, mostrou que a composição e os arranjos dos plantios na região de Tomé-Açu são bastante distintos. Essa distinção de estrutura da vegetação também é evidenciada pelas diferenças encontradas nos índices de diversidade, equabilidade e nos valores de densidade e dominância utilizados no presente trabalho. Estudos de Smith et al. (1998) e de Brienza Júnior et al. (2009) também relataram a existência de grande diversidade de consórcios, praticados por agricultores familiares na Região Amazônica. Os arranjos produtivos na região de ToméAçu potencializaram os SAF como relevante atividade socioeconômica da região, estabelecendo, inclusive, padrões peculiares de uso da terra, com distintos estágios de desenvolvimento vegetativo (Bolfe et al., 2009).

Tabela 4. Índices de diversidade e equabilidade dos sistemas silviagrícolas de Tomé-Açu, PA, 2008.

\begin{tabular}{lccccc}
\hline Indice & SAF 1 & SAF 2 & SAF 3 & SAF 4 & Média \\
\hline Simpson (S') & 0,23 & 0,30 & 0,24 & 0,20 & 0,24 \\
Shannon-Weaver (H') & 1,61 & 1,60 & 1,66 & 2,35 & 1,81 \\
Pielou (J') & 0,73 & 0,54 & 0,56 & 0,64 & 0,62 \\
\hline
\end{tabular}

Tabela 5. Matriz de similaridade florística qualitativa pelo índice de Sörensen $\left(\mathrm{IS}_{\mathrm{s}}\right.$ ) em matriz binária de 54 espécies dos quatro sistemas silviagrícolas (SAF) de Tomé-Açu, PA, 2008.

\begin{tabular}{lllll}
\hline Sistemas silviagrícolas & SAF 1 & SAF 2 & SAF 3 & SAF 4 \\
\hline SAF 1 & & & & \\
SAF 2 & 0,35 & & & \\
SAF 3 & 0,28 & 0,31 & & \\
SAF 4 & 0,20 & 0,40 & 0,51 & \\
\hline
\end{tabular}

Em uma análise mais ampla, é possível relacionar a diversidade e a estrutura observada nos SAF da região de Tomé-Açu com sua capacidade de produção de alimentos, fibras e óleos, e com a potencialidade em acumular carbono na biomassa silviagrícola. Assim, pode-se inferir que os sistemas estudados apresentam peculiaridades intrínsecas a um sistema de produção antrópico, ou seja, aspectos diferenciados quando comparados a outros sistemas silviagrícolas da região.

Os sistemas que integram lavoura-floresta, frequentemente, são admitidos como uma das formas mais adequadas de desenvolvimento dos trópicos úmidos e, junto com o manejo de florestas secundárias (capoeiras) e os reflorestamentos, surgem como alternativas viáveis do ponto de vista ambiental e econômico (Smith et al., 1998). Segundo Osterroht (2002), entre os diversos sistemas agropecuários de uso da terra, os silviagrícolas são aqueles que acumulam o maior ativo de biomassa. A adoção de sistemas de integração de espécies agrícolas e florestais por agricultores familiares é uma estratégia viável e relevante para elevar os estoques de carbono em seus sistemas de produção (Roshetko et al., 2005).

Os resultados obtidos pela análise florística e estrutural dos sistemas estudados, baseados em parâmetros de percentual de cobertura de copa, área basal, altura e idade de implantação média, evidenciaram o estabelecimento de intervalos de classificação para os sistemas silviagrícolas. Estes intervalos permitem definir um sistema de classificação passível de ser testado em outros estudos geoespaciais (Tabela 6).

Tabela 6. Parâmetros e representação esquemática para a classificação de sistemas silviagrícolas.

\begin{tabular}{|c|c|c|c|}
\hline Classificação & $\begin{array}{c}\text { Parâmetros } \\
\text { médios esperados }\end{array}$ & $\begin{array}{l}\text { Cobertura de } \\
\text { copa esperada }\end{array}$ & $\begin{array}{c}\text { Perfil } \\
\text { esperado }\end{array}$ \\
\hline SAF 1 & $\begin{array}{l}\text { Idade: } 0 \text { a } 2 \text { anos } \\
\text { Área basal: } 0 \text { a } 5 \mathrm{~m}^{2} \mathrm{ha}^{-1} \\
\text { Cobertura de copa: } 0 \text { a } 35 \% \\
\text { Altura dos indivíduos: } 0 \text { a } 3 \mathrm{~m}\end{array}$ & & \\
\hline SAF 2 & $\begin{array}{l}\text { Idade: } 2 \text { a } 5 \text { anos } \\
\text { Área basal: } 5 \text { a } 10 \mathrm{~m}^{2} \mathrm{ha}^{-1} \\
\text { Cobertura de copa: } 35 \text { a } 50 \% \\
\text { Altura dos indivíduos: } 3 \text { a } 5 \mathrm{~m}\end{array}$ & & \\
\hline SAF 3 & $\begin{array}{c}\text { Idade: } 5 \text { a } 12 \text { anos } \\
\text { Área basal: } 10 \text { a } 30 \mathrm{~m}^{2} \mathrm{ha}^{-1} \\
\text { Cobertura de copa: } 50 \text { a } 70 \% \\
\text { Altura dos indivíduos: } 4,5 \text { a } 6 \mathrm{~m}\end{array}$ & & \\
\hline SAF 4 & $\begin{array}{l}\text { Idade: acima de } 12 \text { anos } \\
\text { Área basal: acima de } 30 \mathrm{~m}^{2} \mathrm{ha}^{-1} \\
\text { Cobertura de copa: }>70 \% \\
\text { Altura dos indivíduos: }>7 \mathrm{~m}\end{array}$ & & \\
\hline
\end{tabular}

Pesq. agropec. bras., Brasília, v.46, n.10, p.1139-1147, out. 2011 
Assim, a classificação obtida (SAF 1, SAF 2, SAF 3, SAF 4) permite a discriminação entre estágios, apesar da elevada diversidade florística e da baixa similaridade entre os diferentes arranjos produtivos em comparação com outros SAF. Esses dados estão de acordo com Yamada \& Gholz (2002) que observaram que os sistemas silviagrícolas encontrados em Tomé-Açu são uma alternativa de exploração em relação à conversão de florestas em pastagens.

\section{Conclusões}

1. As análises estrutural e florística da vegetação, nos arranjos produtivos dos sistemas silviagrícolas em Tomé-Açu indicam, pelo índice de valor de importância, que as principais espécies componentes são o cacau, o açaí e o cupuaçu.

2. A baixa similaridade florística entre os sistemas silviagrícolas estudados indica elevada heterogeneidade dos arranjos produtivos.

3. As análises estrutural e florística permitem gerar um sistema de classificação para diferenciar os estágios dos sistemas silviagrícolas, passível de utilização em outros estudos.

4. Os sistemas silviagrícolas estudados constituem um sistema de produção diferenciado no contexto da produção agropecuária da Amazônia, considerandose a estrutura e as potencialidades de produção diversificada.

\section{Referências}

BATISTELLA, M.; MORAN, E.F.; ALVES, D.S. Amazônia: natureza e sociedade em transformação. São Paulo: Edusp, 2008. 304p.

BOLFE, E.L.; FERREIRA, M.C.; BATISTELLA, M. Biomassa epígea e estoque de carbono de agroflorestas em Tomé-Açu, PA. Revista Brasileira de Agroecologia, v.4, p.2171-2175, 2009.

BRIENZA JÚNIOR, S.; MANESCHY, R.Q.; MOURÃO JÚNIOR, M.; GAZEL FILHO, A.B.; YARED, J.A.G.; GONÇALVES, D.; GAMA, M.B. Sistemas agroflorestais na Amazônia brasileira: análise de 25 anos de pesquisas. Pesquisa Florestal Brasileira, v.60, p.67-76, 2009.

BROWER, J.E.;ZAR, J.H.; ENDE, C.N. von. Field and laboratory methods for general ecology. New York: WCB/McGraw-Hill, 1998. v.4, 273p.

CARVALHO, J.E.U. de. Utilização de espécies frutíferas em sistemas agroflorestais na Amazônia. In: GAMA-RODRIGUES, A.C. da; BARROS, N.F. de; GAMA-RODRIGUES, E.F. da; FREITAS, M.S.M.; VIANA, A.P.; JASMIN, J.A.; MARCIANO, C.R.; CARNEIRO, J.G. de A. (Ed.). Sistemas agroflorestais: bases científicas para o desenvolvimento sustentável. Campos dos
Goytacazes: Universidade Estadual do Norte Fluminense, 2006. p.169-176.

CASTRO, A.P. de; FRAXE, T. de J.P.; SANTIAGO, J.L.; MATOS, R.B.; PINTO, I.C. Os sistemas agroflorestais como alternativa de sustentabilidade em ecossistemas de várzea no Amazonas. Acta Amazonica, v.39, p.279-288, 2009.

FORZZA, R.C.; LEITMAN, P.M.; COSTA, A.; CARVALHO JUNIOR, A.A. de; PEIXOTO, A.L.; WALTER, B.M.T.; BICUDO, C.; ZAPPI, D.; COSTA, D.P.; LLERAS, E.; MARTINELLI, G.; LIMA, H.C.; PRADO, J.; STEHMANN, J.R.; BAUMGRATZ, J.F.A.; PIRANI, J.R.; SYLVESTRE, L. da S.; MAIA, L.C.; LOHMANN, L.G.; PAGANUCCI, L.; SILVEIRA, M.; NADRUZ, M.; MAMEDE, M.C.H.; BASTOS, M.N.C.; MORIM, M.P.; BARBOSA, M.R.; MENEZES, M.; HOPKINS, M.; SECCO, R.; CAVALCANTI, T.; SOUZA, V. de C. Lista de espécies da flora do Brasil. 2010. Disponível em: <http://floradobrasil.jbrj.gov. br/2010/>. Acesso em: 08 abr. 2011.

GODFREY, B.J.; BROWDER, J.O. Disarticulated urbanization in the Brazilian Amazon. The Geographical Review, v.86, p.441445, 1996.

HOMMA, A.K.O. (Ed.). Amazônia: meio ambiente e desenvolvimento agrícola. Brasília: Embrapa-SPI; Belém: Embrapa-CPATU, 1998. 412p.

HOMMA, A.K.O. (Ed.). História da agricultura na Amazônia: da era pré-colombiana ao terceiro milênio. Belém: Embrapa Amazônia Oriental Brasília; Embrapa Informação Tecnológica, 2003. 274p.

HOMMA, A.K.O. Dinâmica dos sistemas agroflorestais: o caso da Colônia Agrícola de Tomé-Açu, Pará. Revista Instituto de Estudos Superiores da Amazônia, v.2, p.57-65, 2004.

INSTITUTO BRASILEIRO DE GEOGRAFIA E ESTATÍSTICA. Microrregiões do Brasil. 2008. Disponível em: <http://www.ibge. gov.br>. Acesso em: 10 mar. 2008.

KATO, O.R.; KATO, M. do S.A.; CARVALHO, C.J.R. de; FIGUEIREDO, R. de O.; CAMARÃO, A.; SÁ, T.D. deA.; DENICH, M.; VIELHAUER, K. Uso de agroflorestas no manejo de florestas secundárias. In: GAMA-RODRIGUES, A.C. da; BARROS, N.F. de; GAMA-RODRIGUES, E.F. da; FREITAS, M.S.M.; VIANA, A.P.; JASMIN, J.A.; MARCIANO, C.R.; CARNEIRO, J.G. de A. (Ed.). Sistemas agroflorestais: bases científicas para o desenvolvimento sustentável. Campos dos Goytacazes: Universidade do Norte Fluminense, 2006. p.119-138.

LUIZÃO, F.J.; TAPIA-CORAL, S.; GALLARDO-ORDINOLA, J.; SILVA, G.C.; LUIZÃO, R.C.C.; TRUJILLO-CABRERA, L.; WANDELLI, E.; FERNANDES, E.C.M. Ciclos biogeoquímicos em agroflorestas na Amazônia. In: GAMA-RODRIGUES, A.C. da; BARROS, N.F. de; GAMA-RODRIGUES, E.F. da; FREITAS, M.S.M.; VIANA, A.P.; JASMIN, J.A.; MARCIANO, C.R.; CARNEIRO, J.G. de A. (Ed.). Sistemas agroflorestais: bases científicas para o desenvolvimento sustentável. Campos dos Goytacazes: Universidade do Norte Fluminense, 2006. p.87-100.

MAGURRAN, A.E. Ecological diversity and its measurements. Princeton: Princeton University Press, 1988. 179p. 
MENDES, F.A.T. Avaliação de modelos simulados de sistemas agroflorestais em pequenas propriedades cacaueiras selecionadas no Município de Tomé-Açu, no Estado do PA. Informe Gepec, v.7, p.118-144, 2003.

NOBRE, F.R.C. Agroforestry systems in Acre, Brazil: variability in local perspectives. 1998. 172p. Dissertação (Mestrado) University of Florida, Gainesville.

OSTERROHT, M. Princípios filosóficos dos SAF. Agroecologia Hoje, v.3, p.4-19, 2002.

PACHECO, N.A.; BASTOS, T.X. Boletim agrometeorológico 2006: Tomé-Açu, PA. Belém: Embrapa Amazônia Oriental, 2008. 30p.

RIBEIRO, R.N. da S.; TOURINHO, M.M.; SANTANA, A.C. de. Avaliação da sustentabilidade agroambiental de unidades produtivas agroflorestais em várzeas flúvio marinhas de Cametá Pará. Acta Amazonica, v.34, p.359-374, 2004.

RODRIGUES, T.E.; SANTOS, P.L. dos; ROLIM, P.A.M.; SANTOS, E.; REGO, R.S.; SILVA, J.M.L. da; VALENTE, M.A.; GAMA, J.R.N.F. Caracterização e classificação dos solos do Município de Tomé-Açu, Pará. Belém: Embrapa Amazônia Oriental, 2001. 49p.

ROSHETKO, J.M.; LASCO, R.D.; ANGELES, M.S.D. Smallholder agroforestry systems for carbon storage. Mitigation and adaptation strategies for global change, v.12, p.219-242, 2005.

SANTIAGO, J.L. Sistemas agroflorestais tradicionais e a sustentabilidade social das comunidades ribeirinhas do Estado do Amazonas. In: CONGRESSO BRASILEIRO DE
SISTEMAS AGROFLORESTAIS, 5., 2004. Curitiba. SAFs: desenvolvimento com proteção ambiental: anais. Curitiba: Embrapa Florestas, 2004. p.96-104. (Embrapa Florestas. Documentos, 98).

SANTOS, S.R.M. dos; MIRANDA, I.S.; TOURINHO, M.M. Análise florística e estrutural de $\mathrm{SAF}^{\prime} \mathrm{s}$ das várzeas do rio Jubá, Cametá, Pará. Acta Amazônica, v.34, p.251-263, 2004.

SHANNON, C.E. A mathematical theory of communication. Bell System Technical Journal, v.27, p.379-423, 1948.

SMITH, N.; DUBOIS, J.; CURRENT, D.; LUTZ, E.; CLEMENT, C. Experiências agroflorestais na Amazônia brasileira: restrições e oportunidades. Brasília: Programa Piloto para a Proteção das Florestas Tropicais do Brasil, 1998. 146p.

THE ANGIOSPERM PHYLOGENY GROUP. An update of the Angiosperm Phylogeny Group classification for the orders and families of flowering plants: APG III. Botanical Journal of the Linnean Society, v.161, p.105-121, 2009.

VALOIS, A.C.C. Benefícios e estratégias de utilização sustentável da Amazônia. Brasília: Embrapa Informação Tecnológica, 2003. $75 \mathrm{p}$.

VIEIRA, T.A.; ROSA, L. dos S.; VASCONCELOS, P.C.S.; SANTOS, M.M. dos; MODESTO, R. da S. Sistemas agroflorestais em áreas de agricultores familiares em Igarapé-Açu, Pará. Acta Amazonica, v.37, p.549-557, 2007.

YAMADA, M.; GHOLZ, H.L. An evaluation of agroforestry systems as a rural development option for the Brazilian Amazon. Agroforestry Systems, v.55, p.81-87, 2002.

Recebido em 29 de junho de 2010 e aprovado em 25 de agosto de 2011 